Research Paper

\title{
Reduced survival of young patients under 55 years with metastatic prostate cancer: a population-based study
}

\author{
Pan Song ${ }^{1 *}$, Zhufeng Peng1 ${ }^{*}$, Mengxuan Shu ${ }^{2 *}$, Jiaxiang Wang ${ }^{2}$, Peiwen Liu ${ }^{2}$, Xiaoyu Di ${ }^{3}$ Luchen Yang ${ }^{1}$, \\ Zhenghuan Liu $^{1}$, Jing Zhou ${ }^{1}$, Qiang Dong ${ }^{1 凶}$ \\ 1. Department of Urology, West China Hospital of Sichuan University, Chengdu, 610000, Sichuan Province, China. \\ 2. The first Clinical Medical College of Lanzhou University, Lanzhou, 730000, Gansu Province, China. \\ 3. The second Clinical Medical College of Lanzhou University, Lanzhou, 730000, Gansu Province, China. \\ *These authors contributed equally to this study. \\ $\triangle$ Corresponding author: Qiang Dong, Department of Urology, Institute of Urology, West China Hospital of Sichuan University, No. 37, Guoxue Lane, Wuhou \\ District, Chengdu, 610041, China. E-mail: dqiang666@163.com.
}

(1) The author(s). This is an open access article distributed under the terms of the Creative Commons Attribution License (https://creativecommons.org/licenses/by/4.0/). See http:/ /ivyspring.com/terms for full terms and conditions.

Received: 2021.01.13; Accepted: 2021.05.30; Published: 2021.06.11

\begin{abstract}
Objective: The aim of this study was to evaluate the prognosis of patients with metastatic prostate cancer ( $\mathrm{mPCa})$ in different age groups.

Methods: Patients with mPCa from 2004 to 2016 in the Surveillance, Epidemiology and End Results (SEER) database were identified. Seven groups were divided according to the age at diagnosis, including $\leq 55$ years, $56-60$ years, $61-65$ years, $66-70$ years, $71-75$ years, $76-80$ years and $>80$ years. Fine and Gray's competing risks model and Kaplan-Meier analysis were conducted to evaluate the cancer-specific survival (CSS).

Results: A total of 36231 patients with $\mathrm{mPCa}$ were included. The CSS curves of the overall cohort showed that patients aged $\leq 55$ years had significantly worse CSS than patients in age groups of 56-60 [HR:0.93 (0.87 1.00), $p=0.039$ ], $61-65$ [HR:0.91 (0.85 0.97), $p=0.003$ ] and 66-70 [HR:0.90 (0.84 0.96), $\mathrm{p}=0.001$ ]. After removing patients dead for other reasons, the differences of CSS curves between $\leq 55$ years group and $56-70$ years groups were not significant. However, the mean survival time of $\leq 55$ years group (55.78 \pm 2.48 months) was still shorter than 56-60 years (57.28 \pm 2.35 months), 61-65 years (57.64 \pm 2.07 months), and 66-70 years ( $57.11 \pm 2.11$ months). When stratified by $M$ stages, similar results were found in $\mathrm{Mla}, \mathrm{Mlb}$ and Mlc stage groups. According to Fine-Gray competing risks models, patient $\leq 55$ years featured significantly higher sub-distribution hazard ratio (sdHR) than 61-65 years group [sdHR: 0.94(0.88 1.00); $\mathrm{p}=0.046]$.

Conclusions: The mPCa patients $\leq 55$ years seemed to be associated with worse prognosis in comparison with patients aging $56-70$ years.
\end{abstract}

Key words: metastatic prostate cancer; young patients; prognosis, SEER

\section{Introduction}

Prostate cancer (PCa) is the most common malignant tumor in the male genitourinary system, seriously threatening the life and health of men $[1,2]$. By 2020, it was estimated that more than 191,930 men would be newly diagnosed with PCa and 33,330 cases would be dead for PCa [3]. Although there are some radical treatments with excellent long-time prognosis for localized prostate cancer, the prognosis of men with metastatic prostate cancer (mPCa) were rather poor. The common metastatic sites of prostate cancer include bones, distant lymph nodes, liver, and thorax lumbar spine [4, 5]. Metastasis will occur in approximately 15-33\% initial PCa diagnosis within 2 years and has a significant influence on mortality among the population [6]. Androgen deprivation therapy (ADT) is the first-line treatment for men with metastatic prostate cancer, which is usually associated with a good effect at the beginning of treatment $[7,8]$. 
The majority of patients with $\mathrm{MPCa}$ will progress to castration-resistant prostate cancer (CRPC) despite intensive ADT. Once this disease progresses to the castration-resistant stage, the patients are under a substantially great risk of mortality with a median survival time of 15 months [9].

Although the overall prognosis of mPCa patients is rather poor, the survival time of each individual is quite different. The detailed survival time of patients is affected by many factors, such as age, ethnicity, genetic factors etc. [10, 11]. In addition to tumor factors, age is one of the most important factors that has great effects on the prognosis of men with $\mathrm{mPCa}$ [10]. The incidence of prostate cancer is highly correlated with old age. PCa is very rare among young patients and might be more aggressive in terms of biological behavior. Zheng et al. [11] reported that younger patients were associated with significantly worse outcomes in high-risk group patients. For young patients with $\mathrm{mPCa}$, the detailed survival outcomes remain inconclusive.

In this study, we aimed to analyze the prognosis of $\mathrm{mPCa}$ patients in different age groups, and to compare the survival outcomes of young patients with patients of other ages.

\section{Materials and methods}

\section{Data source}

The data of this study were extracted from the SEER database from January 1, 2004, to December 31, 2016. Metastatic PCa patients were retrospectively identified with the software of SEER* STAT. The general information and tumor information were collected.

\section{Inclusion and exclusion criteria}

Inclusion criteria: (1) patients were diagnosed with metastatic PCa (M1 stage). (2) The age of patient at diagnosis was clearly known.

Exclusion criteria: (1) Multiple primary cancers; (2) Important information such as age at diagnosis, $M$ stage, and follow-up time was unknown; (3) The survival status and causes of deaths were unclear at the end of follow up.

\section{Variables and outcomes}

According to the age of patients, we divided the included patients into seven groups: $\leq 55$ years, $56-60$ years, $61-65$ years, 66-70 years, $71-75$ years, $76-80$ years and $>80$ years.

The main outcome in this study was defined as cancer-specific survival (CSS). The survival time was from the patient's first diagnosis to the patient's death or the last follow-up time (December 31, 2016).

\section{Statistical analyses}

Baseline characteristics in seven age groups were presented with frequency and percentage or median and interquartile range (IQR). The Kaplan-Meier (K-M) analysis was conducted to construct the survival curves, and to calculate survival time and survival rates. Hazard ratios (HRs) and their 95\% CIs were used to calculate the degree of risk between different age groups. In order to eliminate the interference of death caused by other reasons except prostate cancer-specific death, we re-analyzed the survival outcomes of the cohort without patients dead for other reasons. The competing risks model with Fine-Gray analysis was also introduced into our analysis. The Fine and Gray's competing risks model provides a useful alternative to Cox regression in the presence of one or more competing risks. It focuses on the cumulative incidence function, which indicates the probability of the event of interest happening before a given time. Subdistribution hazard ratio (sdHR) was calculated with their 95\% CI in this model. All statistical analyses in this study were performed with the software of SPSS version 25, Graphprism 7.2 and STATA 14.0. $\mathrm{P}<0.05$ was considered statistically significant.

\section{Results}

\section{Patient characteristics}

A total of 36231 patients were included. 2712, $3414,4839,5118,5168,5154$ and 9826 patients were in age group of $\leq 55$ years, $56-60$ years, $61-65$ years, $66-70$ years, 71-75 years, 76-80 years and $>80$ years, respectively. The baseline characteristics of seven groups were summarized in Table 1.

\section{Survival analysis}

Survival curves and survival time of the overall cohort

The survival curves of the overall cohort were presented in Figure 1A, patients in $\leq 55$ years group had significantly worse CSS outcomes than those in $56-60$ years group [HR: 0.929, 95\% CI $(0.867 \sim 0.996)$, $\mathrm{p}=0.039]$, 61-65 years group [HR: $0.905,95 \%$ CI (0.847 0.967), $\mathrm{p}=0.003$ ] and 66-70 years group [HR: $0.897,95 \%$ CI $(0.839 \sim 0.958), \mathrm{p}=0.001]$. There was no significant difference between $\leq 55$ years group and 71-75 years group [HR: $0.969,95 \%$ CI $(0.907 \sim 1.034)$, $\mathrm{p}=0.339$ ] and $76-80$ years group [HR: $1.057,95 \% \mathrm{CI}$ (0.991 1.128), $p=0.094]$. The group of age $>80$ years reported the worst survival outcomes.

Considering the difference of metastatic extensions in the included patients, subgroup analyses based on different $\mathrm{M}$ stages were performed. In M1b, significant statistical differences were detected in the comparison of $\leq 55$ vs $56-60$ years 
group [HR: $0.90,95 \% \mathrm{CI}(0.84 \sim 0.94), \mathrm{p}<0.001], \leq 55$ vs 61-65 [HR: $0.87,95 \%$ CI $(0.82 \sim 0.91), \mathrm{p}<0.001], \leq 55$ vs 66-70 [HR: 0.86, 95\% CI (0.82 0.90), p<0.001] and $\leq 55$ vs $71-75$ [HR: $0.93,95 \%$ CI $(0.88 \sim 0.97), p=0.003$ ], individually. 66-70 years group in M1c also presented an apparently better survival outcome than $\leq 55$ group with an HR of 0.87 [95\% CI (0.76 1.00), p=0.037]. No significantly differences were found in the subgroup of M1a group. Detailed results were shown in Figure 1B-D.

Table 1. Baseline characteristics of included patients in overall cohort

\begin{tabular}{|c|c|c|c|c|c|c|c|c|c|}
\hline Characteristic & Total & $\leq 55$ years & $56-60$ years & 61-65 years & $66-70$ years & 71-75 years & 76-80 years & $>80$ yeras & $\mathrm{p}$ \\
\hline $\mathrm{N}$ & 36231 & 2712 & 3414 & 4839 & 5118 & 5168 & 5154 & 9826 & \\
\hline \multicolumn{10}{|l|}{ Year, n (\%) } \\
\hline 2004-2008 & 11688 (32.3) & $894(33)$ & $1111(32.5)$ & $1365(28.2)$ & $1511(29.5)$ & $1653(32)$ & $1823(35.4)$ & $3331(33.9)$ & $<0.001$ \\
\hline 2009-2012 & $10639(29.4)$ & $866(31.9)$ & $980(28.7)$ & $1544(31.9)$ & 1519 (29.7) & $1455(28.2)$ & $1465(28.4)$ & $2810(28.6)$ & \\
\hline 2013-2016 & $13904(38.4)$ & $952(35.1)$ & $1323(38.8)$ & 1930 (39.9) & $2088(40.8)$ & 2060(39.9) & $1866(36.2)$ & $3685(37.5)$ & \\
\hline \multicolumn{10}{|l|}{ Race, n (\%) } \\
\hline White & $27377(75.6)$ & $1818(67)$ & 2307 (67.6) & 3335 (68.9) & 3722 (72.7) & $3890(75.3)$ & $4073(79)$ & $8232(83.8)$ & $<0.001$ \\
\hline Black & $6359(17.6)$ & $758(27.9)$ & $905(26.5)$ & $1175(24.3)$ & 1017 (19.9) & $870(16.8)$ & $689(13.4)$ & $945(9.6)$ & \\
\hline Others & 2495 (6.9) & $136(5)$ & $202(5.9)$ & $329(6.8)$ & $379(7.4)$ & $408(7.9)$ & $392(7.6)$ & 649 (6.6) & \\
\hline \multicolumn{10}{|l|}{ Marriage, n (\%) } \\
\hline Married & $20103(55.5)$ & 1307 (48.2) & $1652(48.4)$ & $2524(52.2)$ & $2958(57.8)$ & $3064(59.3)$ & $3099(60.1)$ & $5499(56)$ & $<0.001$ \\
\hline Unmarried & $5822(16.1)$ & $833(30.7)$ & $935(27.4)$ & $1083(22.4)$ & $846(16.5)$ & $736(14.2)$ & $602(11.7)$ & $787(8)$ & \\
\hline Separated & $7826(21.6)$ & $385(14.2)$ & $590(17.3)$ & $856(17.7)$ & $944(18.4)$ & 972(18.8) & $1112(21.6)$ & $2967(30.2)$ & \\
\hline Unclear & $2480(6.8)$ & $187(6.9)$ & $237(6.9)$ & $376(7.8)$ & $370(7.2)$ & $396(7.7)$ & $341(6.6)$ & $573(5.8)$ & \\
\hline \multicolumn{10}{|l|}{ Grade, n (\%) } \\
\hline Well differentiation & $165(0.5)$ & $16(0.6)$ & $14(0.4)$ & $31(0.6)$ & $25(0.5)$ & $29(0.6)$ & $28(0.5)$ & $22(0.2)$ & $<0.001$ \\
\hline Moderate differentiation & $1856(5.1)$ & $154(5.7)$ & $197(5.8)$ & $282(5.8)$ & $333(6.5)$ & $301(5.8)$ & $258(5)$ & $331(3.4)$ & \\
\hline Poor differentiation & $20539(56.7)$ & $1797(66.3)$ & $2272(66.5)$ & $3181(65.7)$ & $3234(63.2)$ & $3202(62)$ & $2862(55.5)$ & $3991(40.6)$ & $<0.001$ \\
\hline Undifferentiated & $302(0.8)$ & $29(1.1)$ & $30(0.9)$ & $37(0.8)$ & $45(0.9)$ & $33(0.6)$ & $52(1)$ & $76(0.8)$ & \\
\hline Unclear & $13369(36.9)$ & $716(26.4)$ & $901(26.4)$ & $1308(27)$ & $1481(28.9)$ & $1603(31)$ & $1954(37.9)$ & $5406(55)$ & \\
\hline \multicolumn{10}{|l|}{ T stage, n (\%) } \\
\hline T1-2 & $17347(47.9)$ & 1358 (50.1) & $1701(49.8)$ & $2511(51.9)$ & $2637(51.5)$ & $2653(51.3)$ & $2536(49.2)$ & $3951(40.2)$ & $<0.001$ \\
\hline T3-4 & $7300(20.1)$ & $687(25.3)$ & 844 (24.7) & 1096 (22.6) & 1119 (21.9) & 1032(20) & $944(18.3)$ & 1578 (16.1) & \\
\hline Unclear & $11584(32)$ & $667(24.6)$ & $869(25.5)$ & $1232(25.5)$ & $1362(26.6)$ & $1483(28.7)$ & $1674(32.5)$ & 4297 (43.7) & \\
\hline \multicolumn{10}{|l|}{$\mathrm{N}, \mathrm{n}(\%)$} \\
\hline No & 17349 (47.9) & 1109 (40.9) & 1525 (44.7) & $2290(47.3)$ & 2509 (49) & $2633(50.9)$ & $2648(51.4)$ & 4635 (47.2) & $<0.001$ \\
\hline N1 & 8307 (22.9) & 963 (35.5) & 1068 (31.3) & $1362(28.1)$ & 1347 (26.3) & $1124(21.7)$ & 948 (18.4) & 1495 (15.2) & \\
\hline Unclear & 10575 (29.2) & $640(23.6)$ & $821(24)$ & $1187(24.5)$ & $1262(24.7)$ & $1411(27.3)$ & $1558(30.2)$ & $3696(37.6)$ & \\
\hline \multicolumn{10}{|l|}{ M, n (\%) } \\
\hline M1a & $1932(5.3)$ & $194(7.2)$ & $243(7.1)$ & $292(6)$ & $308(6)$ & $280(5.4)$ & $250(4.9)$ & 365 (3.7) & $<0.001$ \\
\hline M1b & $24835(68.5)$ & $1839(67.8)$ & $2260(66.2)$ & $3306(68.3)$ & $3459(67.6)$ & $3622(70.1)$ & $3536(68.6)$ & $6813(69.3)$ & \\
\hline M1c & $7664(21.2)$ & $583(21.5)$ & $778(22.8)$ & $1019(21.1)$ & 1107 (21.6) & 993(19.2) & 1091 (21.2) & $2093(21.3)$ & \\
\hline M1,NOS & $1800(5)$ & $96(3.5)$ & 133 (3.9) & $222(4.6)$ & $244(4.8)$ & $273(5.3)$ & $277(5.4)$ & $555(5.6)$ & \\
\hline \multicolumn{10}{|l|}{ PSA, n (\%) } \\
\hline$<20.0 \mathrm{ng} / \mathrm{ml}$ & 5919 (16.3) & 362 (13.3) & $529(15.5)$ & 779 (16.1) & 1012 (19.8) & $1068(20.7)$ & $943(18.3)$ & $1226(12.5)$ & $<0.001$ \\
\hline $20.0-97.9 \mathrm{ng} / \mathrm{ml}$ & $8287(22.9)$ & $569(21)$ & 759 (22.2) & $1121(23.2)$ & $1183(23.1)$ & $1196(23.1)$ & $1246(24.2)$ & $2213(22.5)$ & \\
\hline$>97.9 \mathrm{ng} / \mathrm{ml}$ & $17158(47.4)$ & $1518(56)$ & $1787(52.3)$ & $2399(49.6)$ & $2338(45.7)$ & $2217(42.9)$ & $2260(43.8)$ & $4639(47.2)$ & \\
\hline Unclear & $4867(13.4)$ & $263(9.7)$ & $339(9.9)$ & $540(11.2)$ & $585(11.4)$ & $687(13.3)$ & $705(13.7)$ & $1748(17.8)$ & \\
\hline \multicolumn{10}{|l|}{ Gleason score, $\mathbf{n}(\%)$} \\
\hline$\leq 7$ & $4728(13)$ & $430(15.9)$ & $528(15.5)$ & $719(14.9)$ & $782(15.3)$ & $771(14.9)$ & $661(12.8)$ & $837(8.5)$ & $<0.001$ \\
\hline $8-10$ & $17327(47.8)$ & $1506(55.5)$ & $1906(55.8)$ & $2713(56.1)$ & $2741(53.6)$ & $2683(51.9)$ & $2414(46.8)$ & $3364(34.2)$ & \\
\hline Unclear & $14176(39.1)$ & $776(28.6)$ & $980(28.7)$ & $1407(29.1)$ & 1595 (31.2) & $1714(33.2)$ & $2079(40.3)$ & $5625(57.2)$ & \\
\hline \multicolumn{10}{|l|}{ Bone metastasis } \\
\hline Yes & $19378(53.5)$ & 1405 (51.8) & $1817(53.2)$ & $2739(56.6)$ & $2871(56.1)$ & $2804(54.3)$ & $2589(50.2)$ & $5153(52.4)$ & $<0.001$ \\
\hline No/Unclear & $16853(46.5)$ & $1307(48.2)$ & $1597(46.8)$ & $2100(43.4)$ & 2247 (43.9) & $2364(45.7)$ & $2565(49.8)$ & $4673(47.6)$ & \\
\hline Lung metastasis & & & & & & & & & 0.596 \\
\hline Yes & $1784(4.9)$ & $129(4.8)$ & $174(5.1)$ & $265(5.5)$ & $253(4.9)$ & $250(4.8)$ & $241(4.7)$ & $472(4.8)$ & \\
\hline No/Unclear & 34447 (95.1) & $2583(95.2)$ & $3240(94.9)$ & $4574(94.5)$ & 4865 (95.1) & 4918(95.2) & 4913 (95.3) & $9354(95.2)$ & \\
\hline Liver metastasis & & & & & & & & & 0.006 \\
\hline Yes & $1007(2.8)$ & $76(2.8)$ & $104(3)$ & $168(3.5)$ & $150(2.9)$ & $147(2.8)$ & $132(2.6)$ & $230(2.3)$ & \\
\hline No/Unclear & $35224(97.2)$ & $2636(97.2)$ & $3310(97)$ & $4671(96.5)$ & 4968 (97.1) & $5021(97.2)$ & $5022(97.4)$ & $9596(97.7)$ & \\
\hline \multicolumn{10}{|l|}{ Brain metastasis } \\
\hline Yes & $255(0.7)$ & $25(0.9)$ & $29(0.8)$ & $46(1)$ & $34(0.7)$ & $33(0.6)$ & $36(0.7)$ & $52(0.5)$ & 0.066 \\
\hline No/Unclear & $35976(99.3)$ & 2687 (99.1) & 3385 (99.2) & 4793 (99) & 5084 (99.3) & $5135(99.4)$ & $5118(99.3)$ & $9774(99.5)$ & \\
\hline \multicolumn{10}{|l|}{ Living status } \\
\hline Alive & $11544(31.9)$ & $1030(38)$ & $1323(38.8)$ & $1937(40)$ & $2049(40)$ & $1818(35.2)$ & 1495 (29) & $1892(19.3)$ & 0.012 \\
\hline Cancer-specific death & $17774(49.1)$ & 1507 (55.6) & $1756(51.4)$ & $2331(48.2)$ & $2333(45.6)$ & $2413(46.7)$ & $2460(47.7)$ & $4974(50.6)$ & \\
\hline Other reason death & $6913(19.1)$ & $175(6.5)$ & $335(9.8)$ & $571(11.8)$ & $736(14.4)$ & $937(18.1)$ & $1199(23.3)$ & $2960(30.1)$ & \\
\hline
\end{tabular}

PSA: prostate-specific antigen. 
(A) Survival curve of age groups in the overall cohort $(P<0.001)$
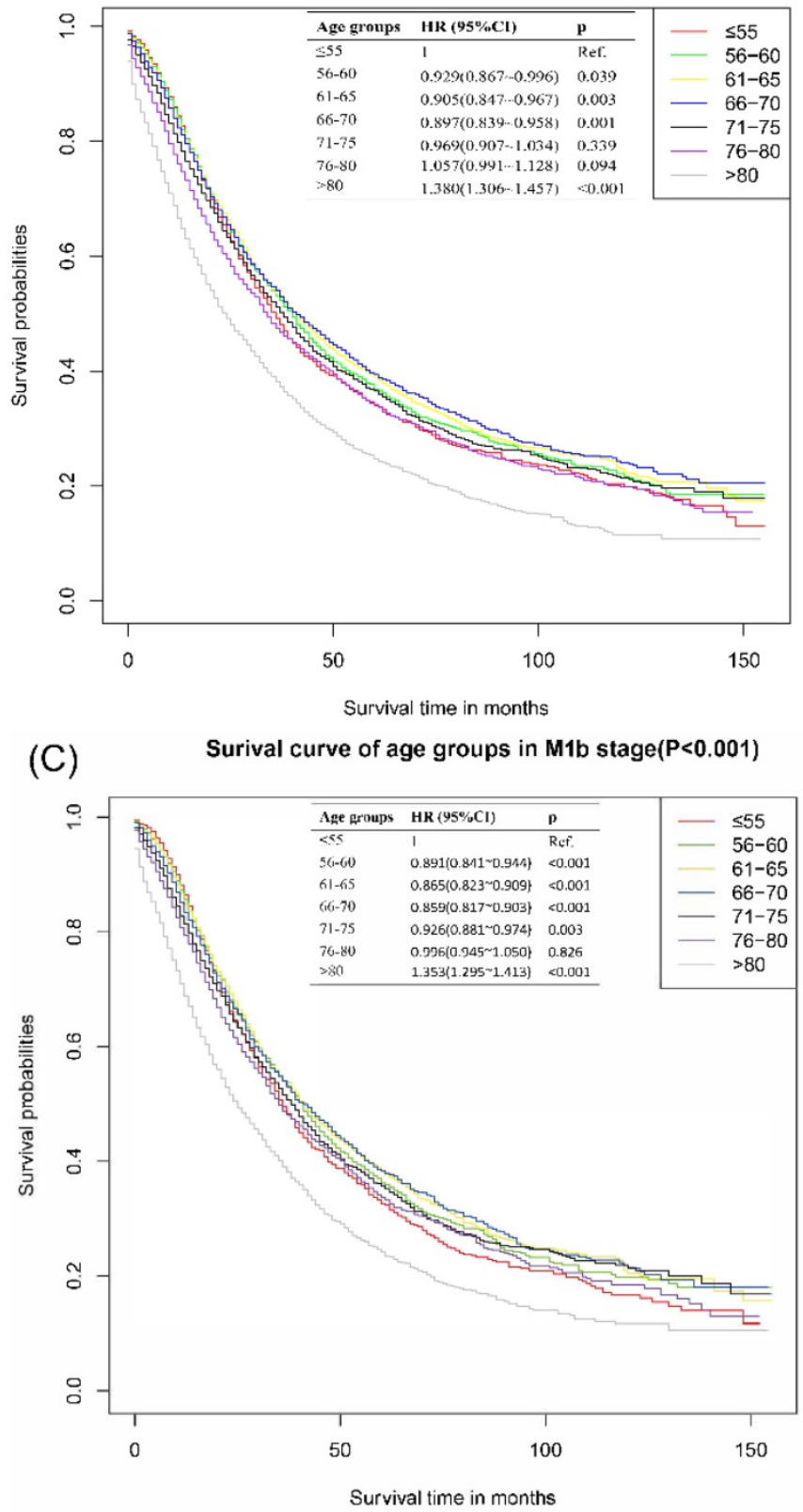

(B)

Surival curve of age groups in M1a stage $(P<0.001)$

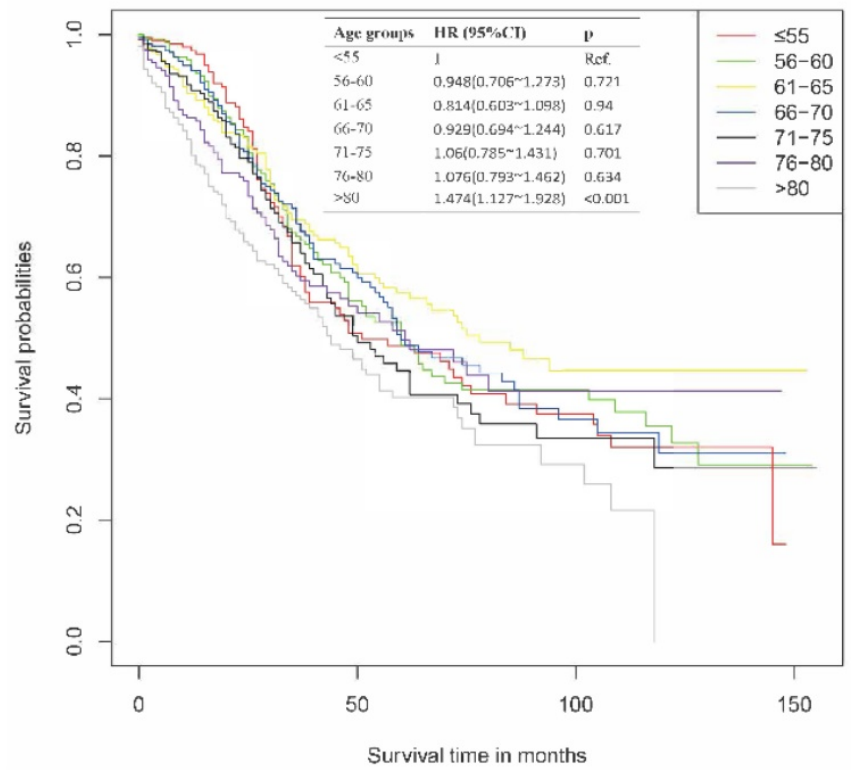

(D)

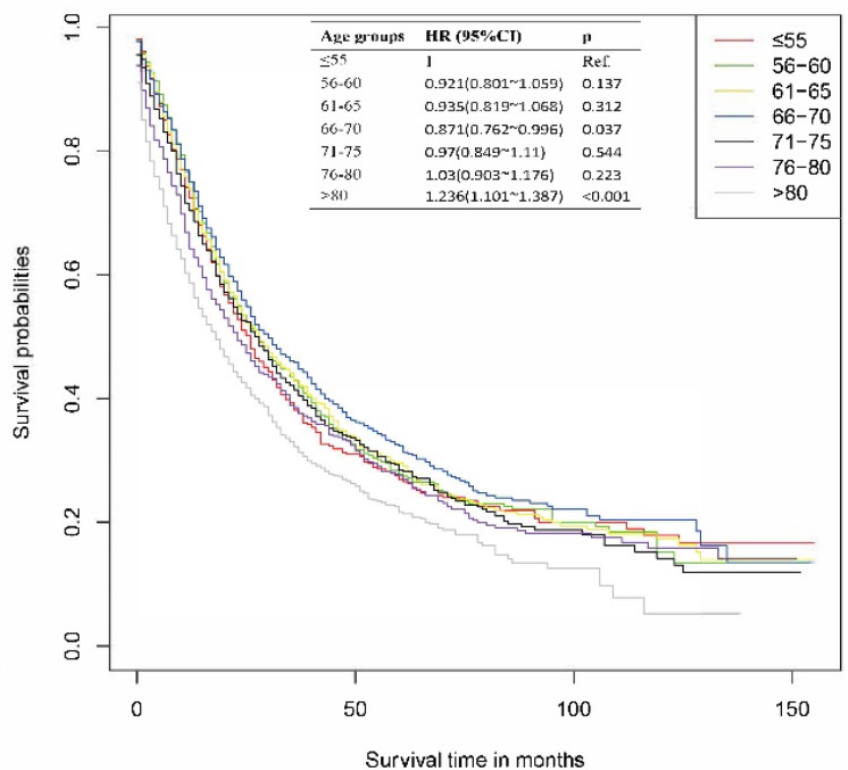

Figure 1. Cancer-specific survival curves of different age groups for patients with metastatic prostate cancer in overall cohort. (A) Cancer-specific survival curves of different age groups for patients with metastatic prostate cancer of all M stages. (B) Cancer-specific survival curves of different age groups for patients with metastatic prostate cancer of Mla stage in overall cohort. (C) Cancer-specific survival curves of different age groups for patients with metastatic prostate cancer of MIb stage in overall cohort. (D) Cancer-specific survival curves of different age groups for patients with metastatic prostate cancer of Mlc stage in overall cohort.

The mean survival time of $\leq 55$ years group was $58.18 \pm 2.52$ months, significantly shorter than $56-60$ years group (61.55 \pm 2.41 months), 61-65 years group (62.99 \pm 2.14 months), 66-70 years group (64.01 \pm 2.2 months) and 71-75 years group (59.87 \pm 2.18 months) with all $\mathrm{M}$ stages. Similar results were found in the subdivided M stage groups. All of the detailed results were presented in Table $\mathbf{2}$. As is also shown in Table 2 , patients $\leq 55$ years had better 1-year survival rate than other age groups. However, The 2-, 3- and 5-year survival rates in $\leq 55$ years groups were significantly worse than those in the age group of 56-60 years,
61-65 years, $66-70$ years and 71-75 years.

Survival curve and survival time of the cohort without patients dead for other reasons

The survival curve of the cohort without patients dead for other reasons was presented in Figure 2A. There were no significant differences among patients $\leq 55$ years, 56-60 years, 61-65 years, and 66-70 years. Patients above 70 years old had worse CSS outcomes than those $\leq 55$ years old. With $\leq 55$ years group as the reference, the $\mathrm{HR}$ and $95 \% \mathrm{CI}$ of patients aged 56-60 years, 61-65 years, 66-70 years, 71-75 years, 76-80 years 
and $>80$ years were $0.97(0.90 \sim 1.03), 0.96(0.90 \sim 1.03)$, 0.98 (0.92 1.05), 1.11 (1.04 1.18), 1.30 (1.22 1.38) and 1.90 (1.81 2.00), respectively. In the subgroup analyses of different $M$ stages, there were no significant differences among $\leq 55$ years, 56-60 years, 61-65 years and 66-70 years groups. These results were presented in Figure 2B-D.

The mean survival time of patients $\leq 55$ years was $55.78 \pm 2.48$ months, which was slightly shorter than those in 56-60 years group (57.28 \pm 2.35 month), 61-65 years group (57.64 \pm 2.07 month), and $66-70$ years group (57.11 \pm 2.11 month). The mean survival time of

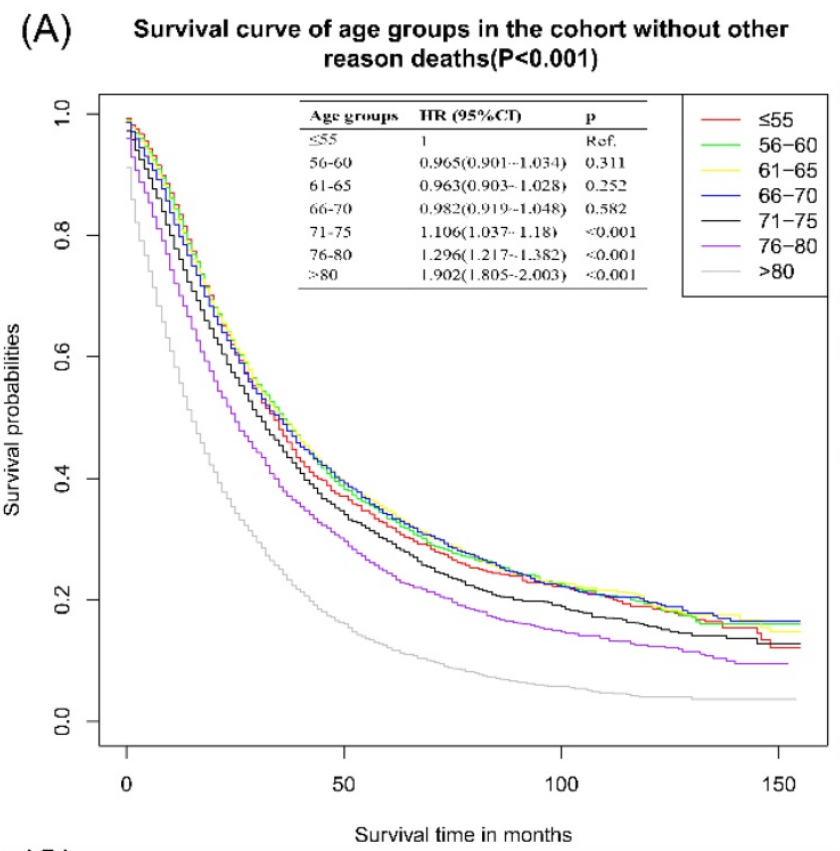

(C) Surival curve of age groups in M1b stage $(P<0.001)$

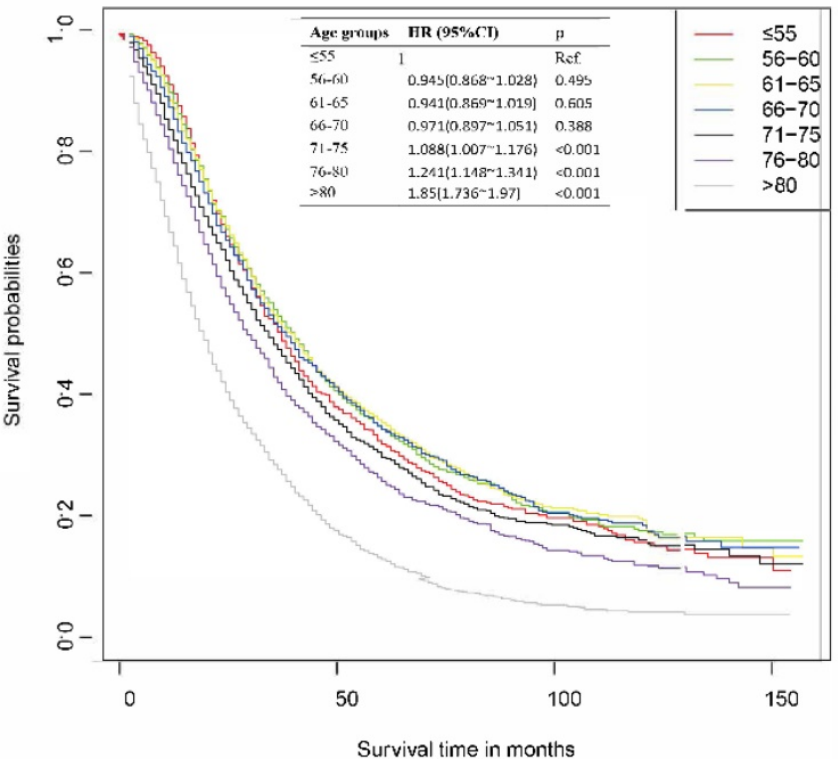

patients aging 76-80 years and $>80$ years were $43.79 \pm 1.89$ month and $27.56 \pm 1.1$ month, individually. Similar results of survival time were found in $\mathrm{M}$ stage subgroups. The survival rate of the cohort without patients dead for other reasons revealed that patients $\leq 55$ years had slightly better 1 -year CSS, similar 2-year CSS, and worse 3- and 5- year CSS than patients in 56-60 years group, 61-65 years group, and 66-70 years group. The survival rate of patients $\leq 55$ years was better than those aging 71-75 years, 76-80 years and $>80$ years. All of the detailed results can be accessed to in Table 2.

\section{(B) Surival curve of age groups in M1a stage $(P<0.001)$}

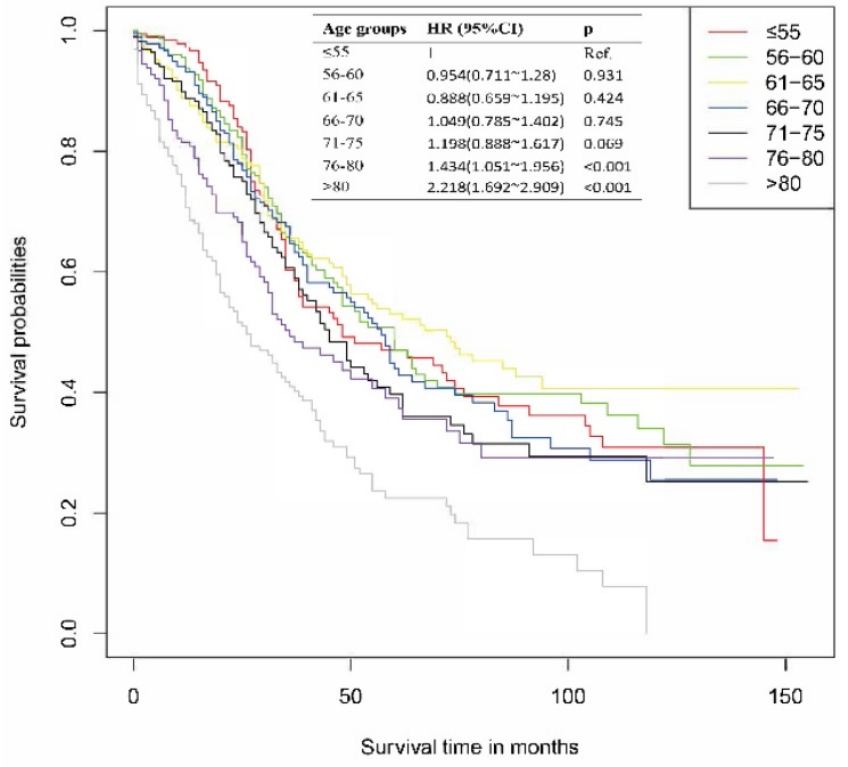

(D) Surival curve of age groups in $M 1 c$ stage $(P<0.001)$

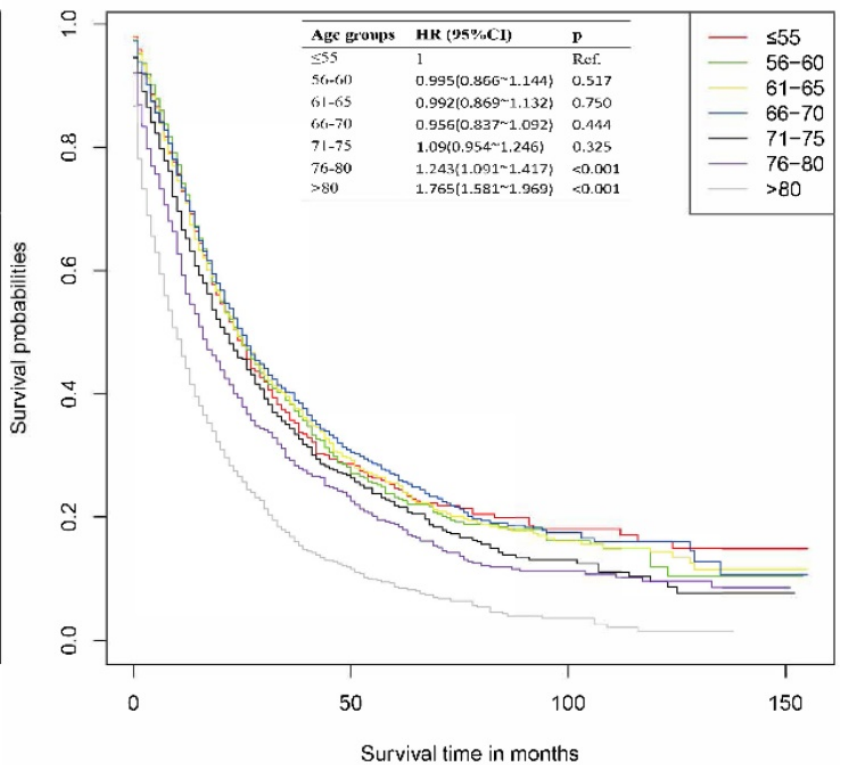

Figure 2. Cancer-specific survival curves of different age groups for patients with metastatic prostate cancer in the cohort that excluded other reason deaths. (A) Cancer-specific survival curves of different age groups for patients with metastatic prostate cancer of all M stages without other-reason death. (B) Cancer-specific survival curves of different age groups for patients with metastatic prostate cancer of Mla without other-reason deaths. (C) Cancer-specific survival curves of different age groups for patients with metastatic prostate cancer of Mlb without other-reason deaths. (D) Cancer-specific survival curves of different age groups for patients with metastatic prostate cancer of Mlc without other-reason deaths. 
Table 2. The cancer-specific survival rates and survival time of patients in different age groups

\begin{tabular}{|c|c|c|c|c|c|c|c|}
\hline CSS & $\leq 55$ years & $56-60$ years & 61-65 years & $66-70$ years & $71-75$ years & $76-80$ years & $>80$ years \\
\hline \multicolumn{8}{|c|}{ Survival time of patients in overall cohort (Mean \pm SD), months } \\
\hline All patients & $58.18 \pm 2.52$ & $61.55 \pm 2.41$ & $62.99 \pm 2.14$ & $64.01 \pm 2.20$ & $59.87 \pm 2.18$ & $55.82 \pm 2.15$ & $43.38 \pm 1.70$ \\
\hline M1a stage & $76.52 \pm 5.00$ & $80.51 \pm 4.80$ & $89.99 \pm 4.71$ & $79.42 \pm 4.49$ & $76.58 \pm 5.47$ & $80.03 \pm 5.46$ & $56.63 \pm 3.4$ \\
\hline M1b stage & $58.28 \pm 1.50$ & $62.19 \pm 1.51$ & $63.85 \pm 1.31$ & $65.16 \pm 1.36$ & $61.56 \pm 1.34$ & $57.22 \pm 1.32$ & $44.4 \pm 1.02$ \\
\hline M1c stage & $50.80 \pm 2.68$ & $53.14 \pm 2.46$ & $52.34 \pm 2.20$ & $55.57 \pm 2.25$ & $48.61 \pm 2.18$ & $47.33 \pm 2.16$ & $35.75 \pm 1.52$ \\
\hline \multicolumn{8}{|c|}{ Survival rates of overall cohort, $\%$} \\
\hline 1- year & $84.0 \%(82.6 \% \sim 85.4 \%)$ & $83.7 \%(82.3 \% \sim 85.1 \%)$ & $82.6 \%(81.4 \% \sim 83.8 \%)$ & $82.0 \%(80.8 \% \sim 83.2 \%)$ & $79.8 \%(78.6 \% \sim 81.0 \%)$ & $76.0 \%(74.8 \% \sim 77.2 \%)$ & $66.7 \%(65.7 \% \sim 67.7 \%)$ \\
\hline 2- year & $63.0 \%(61.0 \% \sim 65.0 \%)$ & $64.6 \%(62.8 \% \sim 66.4 \%)$ & $65.7 \%(64.1 \% \sim 67.3 \%)$ & $64.9 \%(63.3 \% \sim 66.5 \%)$ & $63.1 \%(61.5 \% \sim 64.7 \%)$ & $59.4 \%(57.8 \% \sim 61.0 \%)$ & $49.1 \%(47.9 \% \sim 50.3 \%)$ \\
\hline 3-year & $47.9 \%(45.7 \% \sim 50.1 \%)$ & $52.0 \%(50.0 \% \sim 54.0 \%)$ & $52.5 \%(50.9 \% \sim 54.1 \%)$ & $53.3 \%(51.7 \% \sim 54.9 \%)$ & $50.9 \%(49.3 \% \sim 52.5 \%)$ & $47.4 \%(45.6 \% \sim 49.2 \%)$ & $38.2 \%(37.0 \% \sim 39.4 \%)$ \\
\hline 5- year & $32.7 \%(30.5 \% \sim 34.9 \%)$ & $35.4 \%(33.2 \% \sim 37.6 \%)$ & $37.8 \%(36.0 \% \sim 39.6 \%)$ & $38.4 \%(36.6 \% \sim 40.2 \%)$ & $36.2 \%(34.4 \% \sim 38.0 \%)$ & $34.0 \%(32.2 \% \sim 35.8 \%)$ & $24.7 \%(23.3 \% \sim 26.1 \%)$ \\
\hline \multicolumn{8}{|c|}{ Survival time of the cohort without patients dead for other reasons (Mean $\pm \mathrm{SD}$ ), months } \\
\hline All patients & $55.78 \pm 2.48$ & $57.28 \pm 2.35$ & $57.64 \pm 2.07$ & $57.11 \pm 2.11$ & $50.92 \pm 2.03$ & $43.79 \pm 1.89$ & $27.56 \pm 1.10$ \\
\hline M1a stage & $74.75 \pm 5.01$ & $78.4 \pm 4.81$ & $84.45 \pm 4.84$ & $71.94 \pm 4.53$ & $68.91 \pm 5.40$ & $63.73 \pm 5.65$ & $39.23 \pm 3.06$ \\
\hline M1b stage & $53.28 \pm 1.47$ & $56.50 \pm 1.48$ & $56.81 \pm 1.29$ & $55.66 \pm 1.31$ & $50.70 \pm 1.25$ & $44.47 \pm 1.16$ & $28.26 \pm 0.67$ \\
\hline M1c stage & $46.57 \pm 2.63$ & $44.29 \pm 2.28$ & $45.15 \pm 2.07$ & $46.55 \pm 2.11$ & $39.01 \pm 1.91$ & $34.69 \pm 1.82$ & $20.23 \pm 0.88$ \\
\hline \multicolumn{8}{|c|}{ Survival rates of the cohort without patients dead for other reasons, $\%$} \\
\hline 1- year & $83.0 \%(81.4 \% \sim 84.6 \%)$ & $82.1 \%(80.7 \% \sim 83.5 \%)$ & $80.9 \%(79.7 \% \sim 82.1 \%)$ & $80.5 \%(79.3 \% \sim 81.7 \%)$ & $76.0 \%(74.6 \% \sim 77.4 \%)$ & $69.9 \%(68.3 \% \sim 71.5 \%)$ & $55.7 \%(54.5 \% \sim 56.9 \%)$ \\
\hline 2- year & $61.1 \%(59.1 \% \sim 63.1 \%)$ & $61.8 \%(59.8 \% \sim 63.8 \%)$ & $62.9 \%(61.3 \% \sim 64.5 \%)$ & $62.2 \%(60.6 \% \sim 63.8 \%)$ & $57.2 \%(55.6 \% \sim 58.8 \%)$ & $50.6 \%(48.8 \% \sim 52.4 \%)$ & $35.1 \%(33.9 \% \sim 36.3 \%)$ \\
\hline 3-year & $45.7 \%(43.5 \% \sim 47.9 \%)$ & $48.6 \%(46.6 \% \sim 50.6 \%)$ & $49.4 \%(47.6 \% \sim 51.2 \%)$ & $48.2 \%(46.4 \% \sim 50.0 \%)$ & $44.2 \%(42.4 \% \sim 46.0 \%)$ & $37.7 \%(35.9 \% \sim 39.5 \%)$ & $24.1 \%(22.9 \% \sim 25.3 \%)$ \\
\hline 5- year & $30.7 \%(28.5 \% \sim 32.9 \%)$ & $31.9 \%(29.9 \% \sim 33.9 \%)$ & $34.7 \%(32.9 \% \sim 36.5 \%)$ & $32.9 \%(31.1 \% \sim 34.7 \%)$ & $29.4 \%(27.6 \% \sim 31.2 \%)$ & $24.3 \%(22.5 \% \sim 26.1 \%)$ & $12.1 \%(11.1 \% \sim 13.1 \%)$ \\
\hline
\end{tabular}

Table 3. Multivariate Fine-Gray for patients with metastatic prostate cancer

\begin{tabular}{|c|c|c|}
\hline \multirow[t]{2}{*}{ Risk factors } & \multicolumn{2}{|l|}{ Fine-Gray model } \\
\hline & sdHR $(95 \% \mathrm{CI})$ & $\mathrm{p}$ \\
\hline \multicolumn{3}{|l|}{ Age } \\
\hline$\leq 55$ years & 1 & Ref. \\
\hline $56-60$ years & $0.95(0.89 \sim 1.02)$ & 0.149 \\
\hline 61-65 years & $0.94(0.88 \sim 0.99)$ & 0.046 \\
\hline $66-70$ years & $0.95(0.9 \sim 1.01)$ & 0.130 \\
\hline $71-75$ years & $1.04(0.97 \sim 1.11)$ & 0.262 \\
\hline $76-80$ years & 1.07 (1.00 1.14) & 0.034 \\
\hline$>80$ years & 1.34 (1.27 1.43) & $<0.001$ \\
\hline \multicolumn{3}{|l|}{ Year of diagnosis } \\
\hline 2004-2008 & 1 & Ref. \\
\hline 2009-2012 & $0.91(0.88 \sim 0.94)$ & $<0.001$ \\
\hline 2013-2016 & $0.84(0.81 \sim 0.88)$ & $<0.001$ \\
\hline \multicolumn{3}{|l|}{ Race } \\
\hline White & 1 & Ref. \\
\hline Black & 1.03 (0.99 1.07) & 0.145 \\
\hline Others & $0.79(0.74 \sim 0.84)$ & $<0.001$ \\
\hline \multicolumn{3}{|l|}{ Marriage } \\
\hline Married & 1 & Ref. \\
\hline Unmarried & $1.18(1.13 \sim 1.24)$ & $<0.001$ \\
\hline Separated & 1.18 (1.14 1.23) & $<0.001$ \\
\hline \multicolumn{3}{|l|}{ M } \\
\hline M1a & 1 & Ref. \\
\hline M1b & $1.63(1.51 \sim 1.76)$ & $<0.001$ \\
\hline M1c & 1.97 (1.81 2.13) & $<0.001$ \\
\hline \multicolumn{3}{|l|}{ PSA } \\
\hline$<20.0 \mathrm{ng} / \mathrm{ml}$ & 1 & Ref. \\
\hline $20.0-97.9 \mathrm{ng} / \mathrm{ml}$ & 1.18 (1.12 1.25) & $<0.001$ \\
\hline$\geq 98.0 \mathrm{ng} / \mathrm{ml}$ & $1.47(1.4 \sim 1.55)$ & $<0.001$ \\
\hline \multicolumn{3}{|l|}{ Gleason score } \\
\hline$\leq 7$ & 1 & Ref. \\
\hline $8-10$ & 1.51 (1.43 1.59) & $<0.001$ \\
\hline
\end{tabular}

HR: hazard ratio; sdHR: subdistribution hazard ratio; $95 \%$ CI: 95\% confidence interval; Ref: reference; PSA: prostate-specific antigen.

\section{Multivariate Fine-Gray analysis}

The results of multivariate Fine-Gray analysis in competitive risk model were shown in Table 3. The factors, including age, year of diagnosis, race, marital status, PSA levels and Gleason score, were independent prognostic risk factors. With age $\leq 55$ years as the reference, the sdHR and $95 \% \mathrm{CI}$ of patients aged 56-60 years, 61-65 years, 66-70 years, 71-75 years, 76-80 years and >80 years were 0.95 (0.89 1.02), $0.94 \quad(0.88 \sim 0.99), \quad 0.95 \quad(0.9 \sim 1.01), 1.04$ (0.97 1.11), 1.07 (1.00 1.14) and 1.34 (1.27 1.43), respectively.

\section{Discussion}

Although early-stage PCa can usually be cured by radical surgery, the prognosis for patients with distant metastasis is rather poor even with the optimal treatment. Once prostate cancer reaches the metastatic stage, the average life expectancy of patients will be severely reduced [12]. Age is an important factor which has great effects on the prognosis of individuals. mPCa in young patients seems to be more aggressive and poorly differentiated. The prognosis of young patients with $\mathrm{mPCa}$ has not been evaluated in detail.

Our results found that the survival outcomes of patients $\leq 55$ years were significantly worse than those of patients aged 56-60 years, 61-65 years and 66-70 years, similar to those aging $71-75$ years. These results had been proven by some previous studies. Kimura et al.[13] found the 5-years overall survival rates in the young group (aged $\leq 55$ years) were significantly worse than those in the middle-aged (aged $\geq 56$ and $\leq 65$ years) and elderly groups (aged $\geq 66$ and $\leq 75$ years) in patients with stage IV disease with metastasis. Hamstra et al. [14] revealed that older age was associated with decreased metastasis and prostate cancer-specific death for men with localized advanced prostate cancer. They found that the cancer in older men was less aggressive, and was 
independent of other clinical features. Humphreys et al. [15] also showed a trend towards worse survival in patients aged $<55$ years. On the contrary, some studies found older patients ( $>70$ years or even older) had a poorer prognosis than the younger $[16,17]$. Guo et al. [18] demonstrated that younger patients $(\leq 70$ years old) with bone and lung metastases presented better survival outcomes than those with other types of metastasis or older age.

To avoid the interference caused by other-reason deaths, we reanalyzed the survival outcomes of the cohort without men dead for other reasons and introduced the competitive risk model. Our results reported that young patients ( $\leq 55$ years) still had slightly worse survival outcomes than patients aged 56-60 years, 61-65 years and 66-70 years, especially for patients in 61-65 years group. Similarly, Bernard et al. [19] revealed that men aged $<55$ years presented second worst prognosis with lower median PCSS and a higher cumulative incidence of death due to PCa compared with patients aged 55-64 years and 65-74 years.

Some previous studies accounted for the results in this study. Compared with older mPCa patients, younger patients were associated with more aggressive and much advanced stages, resulting in worse outcomes [20-22]. Though some studies reported more favorable outcomes in younger patients, the differences of detection and screening methods and patient selection may contribute to these discrepancies [23]. Another possible explanation for worse prognosis in the men aged $\leq 55$ years in comparison with the older is that the incidence of BRCA1 and BRCA2 mutation is much higher in young men (aged $<65$ years) [24] and the BRCA1/2 mutation is correlated with poor prognosis. Men with BRCA1/2 mutation were more likely to have a higher Gleason score and worse prognoses than non-BRCA2 carriers.

There were some limitations in our study. First, this was a retrospective analysis along with some unavoidable confounders and risk biases, which may lead to incompletion of clinical information. Second, limited by the data we could obtained, some factors such as detailed treatments, financial situation, etc. were not analyzed in our study. Our results might be influenced by these factors. Therefore, more high-quality studies are needed in the future to verify our results.

\section{Conclusions}

For $\mathrm{mPCa}$, the prognoses of young patients $(\leq 55$ years) were slightly worse than those aged between 56 and70 years. However, considering the limitation in our study, more high-quality studies are needed in the future.

\section{Acknowledgements}

\section{Funding}

This work was supported by a Key Project of National Natural Science Foundation of China; Grant ID: 8177060452; and 1.3.5 project for disciplines of excellence, West China Hospital, Sichuan University, Grant ID: ZY2016104.

\section{Ethical Approval}

All data was from the public database and no ethical approval was required.

\section{Availability of data and materials}

The data for constructing models was obtained from the SEER database which is publicly available.

\section{Competing Interests}

The authors have declared that no competing interest exists.

\section{References}

1. Ferlay J, Soerjomataram I, Dikshit R, Eser S, Mathers C, Rebelo M, et al. Cancer incidence and mortality worldwide: sources, methods and major patterns in GLOBOCAN 2012. International journal of cancer. 2015; 136: E359-86.

2. Torre LA, Siegel RL, Ward EM, Jemal A. Global Cancer Incidence and Mortality Rates and Trends - An Update. Cancer epidemiology, biomarkers \& prevention: a publication of the American Association for Cancer Research, cosponsored by the American Society of Preventive Oncology. 2016; 25: 16-27.

3. Siegel RL, Miller KD, Jemal A. Cancer statistics, 2020. CA: a cancer journal for clinicians. 2020; 70: 7-30.

4. Steele CB, Li J, Huang B, Weir HK. Prostate cancer survival in the United States by race and stage (2001-2009): Findings from the CONCORD-2 study. Cancer. 2017; 123 Suppl 24: 5160-77.

5. Gandaglia G, Abdollah F, Schiffmann J, Trudeau V, Shariat SF, Kim SP, et al. Distribution of metastatic sites in patients with prostate cancer: A population-based analysis. The Prostate. 2014; 74: 210-6.

6. Hirst CJ, Cabrera C, Kirby M. Epidemiology of castration resistant prostate cancer: a longitudinal analysis using a UK primary care database. Cancer epidemiology. 2012; 36: e349-53.

7. Nguyen PL, Alibhai SM, Basaria S, D'Amico AV, Kantoff PW, Keating NL, et al. Adverse effects of androgen deprivation therapy and strategies to mitigate them. European urology. 2015; 67: 825-36.

8. Nead KT, Gaskin G, Chester C, Swisher-McClure S, Leeper NJ, Shah NH. Association Between Androgen Deprivation Therapy and Risk of Dementia. JAMA oncology. 2017; 3: 49-55.

9. de Bono J, Mateo J, Fizazi K, Saad F, Shore N, Sandhu S, et al. Olaparib for Metastatic Castration-Resistant Prostate Cancer. The New England journal of medicine. 2020; 382: 2091-102.

10. Wise HM, Hermida MA, Leslie NR. Prostate cancer, PI3K, PTEN and prognosis. Clinical science (London, England : 1979). 2017; 131: 197-210.

11. Zheng $\mathrm{Y}$, Lin SX, Wu S, Dahl DM, Blute ML, Zhong WD, et al. Clinicopathological characteristics of localized prostate cancer in younger men aged $\leq 50$ years treated with radical prostatectomy in the PSA era: A systematic review and meta-analysis. Cancer medicine. 2020; 9: 6473-84.

12. Mateo J, Fizazi K, Gillessen S, Heidenreich A, Perez-Lopez R, Oyen WJG, et al. Managing Nonmetastatic Castration-resistant Prostate Cancer. European urology. 2019; 75: 285-93.

13. Kimura T, Onozawa M, Miyazaki J, Matsuoka T, Joraku A, Kawai K, et al. Prognostic impact of young age on stage IV prostate cancer treated with primary androgen deprivation therapy. International journal of urology : official journal of the Japanese Urological Association. 2014; 21: 578-83.

14. Hamstra DA, Bae K, Pilepich MV, Hanks GE, Grignon DJ, McGowan DG, et al. Older age predicts decreased metastasis and prostate cancer-specific death for men treated with radiation therapy: meta-analysis of radiation therapy oncology group trials. International journal of radiation oncology, biology, physics. 2011; 81: 1293-301. 
15. Humphreys MR, Fernandes KA, Sridhar SS. Impact of Age at Diagnosis on Outcomes in Men with Castrate-Resistant Prostate Cancer (CRPC). Journal of Cancer. 2013; 4: 304-14.

16. Liu D, Kuai Y, Zhu R, Zhou C, Tao Y, Han W, et al. Prognosis of prostate cancer and bone metastasis pattern of patients: a SEER-based study and a local hospital based study from China. Scientific reports. 2020; 10: 9104.

17. $\mathrm{Hu}$ MB, Yang $\mathrm{T}, \mathrm{Hu} \mathrm{JM}$, Zhu WH, Jiang HW, Ding O. Prognostic factors in Chinese patients with prostate cancer receiving primary androgen deprivation therapy: validation of Japan Cancer of the Prostate Risk Assessment (J-CAPRA) score and impacts of pre-existing obesity and diabetes mellitus. International journal of clinical oncology. 2018; 23: 591-8.

18. Guo Y, Mao S, Zhang A, Wang R, Zhang Z, Zhang J, et al. Prognostic Significance of Young Age and Non-Bone Metastasis at Diagnosis in Patients with Metastatic Prostate Cancer: a SEER Population-Based Data Analysis. Journal of Cancer. 2019; 10: 556-67.

19. Bernard B, Burnett C, Sweeney CJ, Rider JR, Sridhar SS. Impact of age at diagnosis of de novo metastatic prostate cancer on survival. Cancer. 2020; 126 : 986-93.

20. Huben R, Natarajan N, Pontes E, Mettlin C, Smart CR, Murphy GP. Carcinoma of prostate in men less than fifty years old. Data from American College of Surgeons' National Survey. Urology. 1982; 20: 585-8.

21. Johnson DE, Lanieri JP, Jr., Ayala AG. Prostatic adenocarcinoma occurring in men under 50 years of age. Journal of surgical oncology. 1972; 4: 207-16.

22. Sandhu DP, Munson KW, Benghiat A, Hopper IP. Natural history and prognosis of prostate carcinoma in adolescents and men under 35 years of age. British journal of urology. 1992; 69: 525-9.

23. Wu D, Ni J, Beretov J, Cozzi P, Willoox M, Wasinger V, et al. Urinary biomarkers in prostate cancer detection and monitoring progression. Critical reviews in oncology/hematology. 2017; 118: 15-26.

24. Nyberg T, Frost D, Barrowdale D, Evans DG, Bancroft E, Adlard J, et al. Prostate Cancer Risks for Male BRCA1 and BRCA2 Mutation Carriers: A Prospective Cohort Study. European urology. 2020; 77: 24-35. 